\title{
The pharmacokinetics of Tiangou antihypertensive capsule in rat in vivo
}

\author{
JI-ZHENG SONG, LING-JUN LI, LI JI, LI SHUN and YAN RUI \\ College of Pharmacy, Shandong University of Traditional Chinese Medicine, Jinan, Shandong 250355, P.R. China
}

Received July 19, 2016; Accepted September 26, 2016

DOI: $10.3892 /$ br.2016.810

\begin{abstract}
The aim of the study was to examine the effect of complex prescription on the pharmacokinetics of baicalin, rhynochophylline and gastrodin in Tiangou antihypertensive capsule. After administration, rat plasma was collected at different time-points. High-performance liquid chromatography was used to determine the content of baicalin, rhynochophylline and gastrodin in plasma. Two peaks occurred in the baicalin concentration-time curve. No significant difference was found for the peak concentration time $\left(\mathrm{t}_{\max }\right)$ and area under concentration-time curve (AUC) of baicalin between the complex prescription and baicalin groups. The peak concentration $\left(\mathrm{C}_{\max }\right)$ of baicalin in the complex prescription group was significantly decreased, while no significant difference was found for the absorption factor $\left(\mathrm{K}_{\mathrm{a}}\right)$ and AUC of baicalin between the complex prescription and gastrodin groups. The elimination factor $\left(\mathrm{K}_{\mathrm{e}}\right)$ of gastrodin in the complex prescription group was significantly decreased, while the apparent volume of distribution ( $\mathrm{Vd}$ ) of gastrodin was significantly increased. No significant difference was found for AUC of baicalin between the complex prescription and rhynochophylline groups. Levels of $\mathrm{K}_{\mathrm{a}}$ and $\mathrm{K}_{\mathrm{e}}$ of rhynochophylline were lower in the complex prescription group while Vd was higher. Thus, complex prescription made plasma concentration-time curve more smooth. By contrast, the Tiangou antihypertensive capsule improved the distribution of baicalin, rhynochophylline and gastrodin in vivo.
\end{abstract}

\section{Introduction}

Hypertension is a chronic disease, and its major characteristics include increasing arterial blood pressure, which often causes heart, brain, kidney and other vital organ diseases and

Correspondence to: Professor Ling-Jun Li, College of Pharmacy, Shandong University of Traditional Chinese Medicine, University Road no. 4655, Jinan, Shandong 250355, P.R. China

E-mail: 11jun66@163.com

Key words: baicalin, complex prescription, gastrodin, high-performance liquid chromatography, pharmacokinetic, rhynochophylline corresponding consequences $(1,2)$. The Tiangou Jiangya capsule is composed of Gastrodia elata, Uncaria rhynochophylla, Eucommia, Scutellaria baicalensis, Cortex moutan and Pearl, and represses hyperactive liver yang and sedative, which was used to treat mild to moderate hypertension $(1,2)$.

Currently, Chinese herbal monomers are the subject of Traditional Chinese Medicine (TCM) pharmacokinetic studies (3-5). However, as the efficacy of TCM is attributed to the overall effect of various compounds, investigating only one ingredient is not considered to be beneficial to TCM research (6). In the present study, the effective ingredients of the Tiangou antihypertensive capsule were determined. The ingredients were used as a reference value to prepare the equivalent concentration of the extract solution. This solution was then used to examine the effects of complex prescription on concentration-time curve and the pharmacokinetic parameters of baicalin, rhynochophylline and gastrodin. The results provided valuable data for the further study of the Tiangou Jiangya capsule.

\section{Materials and methods}

Instrumentation. Instruments used in the present study were: D2100 Hitachi high-performance liquid chromatography (HPLC), AE240 electronic balance (Mettler-Toledo Instruments Co., Ltd., Shanghai, China), HSC-12A Organomation (Beijing Zhuoxin Weiye Technology Co., Ltd., Beijing, China), Nitrogen (Jinan Dehui Gas Co., Ltd., Jinan, China), XK96-B fast mixer (Jiangsu Xinkang Medical Instrument Co., Ltd., Jiangsu, China), KQ-250E ultrasonic cleaning instrument (Kunshan Ultrasonic Instrument Co., Ltd., Kunshan, China), LDZ4-0.8 medical centrifuge (Beijing Medical Centrifuge Factory, Beijing, China), TGL-16G high speed centrifuge (analysis of Shanghai Medical Instrument Factory, Shanghai, China) and Eppendorf tube (JiNan YongNen Technology Co., Ltd., JiNan, China).

Reagents and chemicals. Reagents used in the present study included, heparin sodium injection (lot no. 10067; Changzhou Qianhong Biochemical Pharmaceutical Co., Ltd., Changzhou, China); chloral hydrate (lot no. 20081021; Tianjin Guangfu Institute Fine Chemical Research, Tianjin, China); acetonitrile (Shandong Yuwang Industrial Co., Ltd. Jinan, China); and methanol (Tianjin Siyou Fine Chemicals Co., Ltd., Tianjin, China). Acetonitrile and methanol were chromatographically 
Table I. Regression equation and LOD of the analytes in rat plasma.

\begin{tabular}{llccc}
\hline Compound & Regression equation & Correlation coefficient $(\mathrm{r})$ & Linear range $(\mu \mathrm{g} / \mathrm{ml})$ & LOD $(\mathrm{ng} / \mathrm{ml})$ \\
\hline Baicalin & $\mathrm{y}=0.052 \mathrm{x}-0.026$ & 0.9995 & $0.44-28.00$ & 1.85 \\
Gastrodin & $\mathrm{y}=0.0219 \mathrm{x}-0.0138$ & 0.9991 & $0.13-13.00$ & 4.31 \\
Rhynochophylline & $\mathrm{y}=0.0091 \mathrm{x}+0.0002$ & 0.9993 & $0.04-2.34$ & 2.92 \\
\hline
\end{tabular}

Table II. Summary of precision, accuracy and recovery in rat plasma $(n=6)$.

\begin{tabular}{|c|c|c|c|c|c|}
\hline Analytes & Concentration $(\mu \mathrm{g} / \mathrm{ml})$ & Intra-day precision (\%) & Inter-day precision $(\%)$ & Accuracy (\%) & Recovery (\%) \\
\hline \multirow[t]{4}{*}{ Baicalin } & 0.87 & - & - & - & 98.85 \\
\hline & 3.62 & 4.80 & 4.03 & 3.4 & 100.08 \\
\hline & 13.72 & - & - & - & 98.73 \\
\hline & 0.13 & - & - & - & 99.77 \\
\hline \multirow[t]{3}{*}{ Gastrodin } & 8.17 & 2.18 & 3.12 & 2.39 & 102.17 \\
\hline & 13.00 & - & - & - & 100.34 \\
\hline & 0.04 & - & - & - & 97.71 \\
\hline \multirow[t]{2}{*}{ Rhynochophylline } & 0.51 & 4.26 & 3.42 & 4.89 & 99.09 \\
\hline & 2.54 & - & - & - & 101.18 \\
\hline
\end{tabular}

pure, while other reagents were analytically pure, and water was Wahaha Purified water (Hangzhou Wahaha Group Co., Ltd., Zhejiang, China).

The chemicals included in the study were: Baicalin reference standard (98\% purity, lot no. 110715-201016; National Institutes for Food and Drug Control, Beijing, China); rutin (98\% purity, lot no. 10080-200707; National Institutes for Food and Drug Control); rhynochophylline (98\% purity, lot no. 20120426 Shanghai Standard Source Leaves Biological Technology Co., Ltd., Shanghai, China); gastrodin (98\% purity, lot no. 110807-200205; National Institutes for Food and Drug Control); and the Tiangou antihypertensive capsule (home made, containing $0.7 \%$ rhynochophylline, $5.6 \%$ gastrodin, $7.9 \%$ baicalin, and $1.2 \%$ baicalein).

Experimental animals. Fifty-four male Sprague-Dawley (SD) (production license no. SCXK-Lu-20130001), with a body weight of $220 \pm 20 \mathrm{~g}$, were purchased from the Experimental Animal Center of Shandong Lukang (Shandong, China). The 54 rats were randomly divided into 9 groups ( $\mathrm{n}=6$ rats/group) labelled A-I. The procedures involving animals and their care were conducted in accordance with NIH guidelines (publication no. 85-23, revised 1996) and were approved by the Animal Care and Use Committee of the Shandong University of Traditional Chinese Medicine.

HPLC. For baicalin, analysis separation was performed on a Shimadzu ODS $(4.6 \times 250 \mathrm{~mm}, 5 \mu \mathrm{m})$ maintained at $25^{\circ} \mathrm{C}$. Methanol-water-phosphoric (47:53:0.2) was used as the mobile phase at $1.0 \mathrm{ml} / \mathrm{min}$. Solution $(20 \mu \mathrm{l})$ was injected into the system. The detector wavelength was $280 \mathrm{~nm}$ (7). Rutin was used as the internal standard substance.

For gastrodin, analysis separation was performed on a Shimadzu ODS $(4.6 \times 250 \mathrm{~mm}, 5 \mu \mathrm{m})$ maintained at $30^{\circ} \mathrm{C}$.
Acetonitrile-0.05\% acid water solution (3:97) was used as the mobile phase at $1.0 \mathrm{ml} / \mathrm{min}$ and $20 \mu \mathrm{l}$ solution was injected into the system. The detector wavelength was $220 \mathrm{~nm}(7)$. Phloroglucinol was used as the internal standard substance.

For rhynochophylline, analysis separation was performed on a Shimadzu ODS $(4.6 \times 250 \mathrm{~mm}, 5 \mu \mathrm{m})$ maintained at $25^{\circ} \mathrm{C}$. Methanol-water (55:45) (containing $0.01 \mathrm{~mol} / \mathrm{l}$ triethylamine, acetic acid adjust solution $\mathrm{pH} 7.5$ ) was used as the mobile phase at $1.0 \mathrm{ml} / \mathrm{min}$. Solution $(20 \mu \mathrm{l})$ was injected into the system, and the detector wavelength was $254 \mathrm{~nm}$ (8).

Preparation of standard and quality control samples. The stock solutions of baicalin, rhynochophylline and gastrodin were prepared in $10 \mathrm{ml}$ methanol at a concentration of $500 \mu \mathrm{g} / \mathrm{ml}$. A series of standard working solutions were prepared by the diluted proper stock solutions with methanol. Calibration working solutions were prepared by adding the diluted working standard solutions into blank rat plasma $(5 / 95, \mathrm{v} / \mathrm{v})$, and the resulting concentrations of baicalin, gastrodin and rhynochophylline are shown in Table I, respectively.

The quality control samples were prepared at three concentrations of $0.87,3.62$ and $13.72 \mu \mathrm{g} / \mathrm{ml}$ for baicalin; $0.13,8.17$ and $13.00 \mu \mathrm{g} / \mathrm{ml}$ for gastrodin; and $0.04,0.51$ and $2.54 \mu \mathrm{g} / \mathrm{ml}$ for rhynochophylline. The solutions were stored at $4^{\circ} \mathrm{C}$ in a refrigerator and brought to room temperature before use. The results are shown in Table II.

Selectivity. Blank plasma spiked with the internal standard and standard substance, and the rat plasma sample collected after oral administration of the Tiangou antihypertensive capsule solution were prepared, respectively. The samples were treated with precipitate protein, and the compounds in plasma were quantitatively determined by the abovementioned analysis methods. The HPLC spectra of plasma samples are 
Table III. Initial oral administration dosage.

\begin{tabular}{lccc}
\hline Groups & $\begin{array}{c}\text { Tiangou anti-hypertensive } \\
\text { capsule (ml) }\end{array}$ & $\begin{array}{c}\text { Scutellaria baicalensis } \\
\text { extract (ml) }\end{array}$ & $\begin{array}{c}\text { Gastrodia Rhizoma } \\
\text { extract (ml) }\end{array}$ \\
\hline Low dose (A) & 1.5 & - & - \\
Mid dose (B) & 3.0 & - & - \\
High dose (C) & 6.0 & - & - \\
Scutellaria control (D) & - & - & - \\
Gastrodia elata control (E) & - & 1.8 \\
\hline
\end{tabular}

Table IV. Oral administration dosage.

\begin{tabular}{lcc}
\hline Group & Baicalin $(\mathrm{ml})$ & Baicalein $(\mathrm{mg})$ \\
\hline $\mathrm{F}$ & 4.0 & - \\
$\mathrm{G}$ & - & 2.4 \\
\hline
\end{tabular}
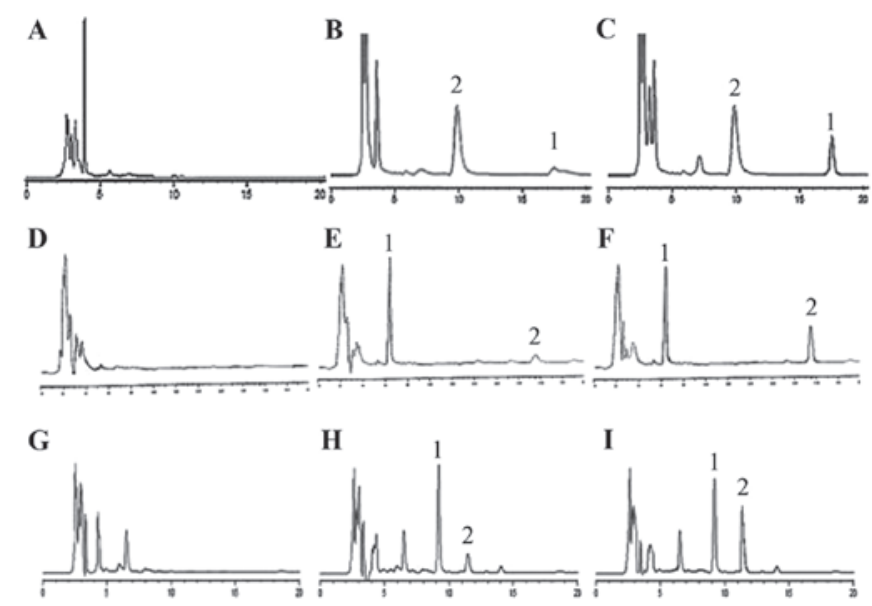

Figure 1. High-performance liquid chromatography spectrum of plasma samples. (A) Blank plasma; (B) 1, baicalin; 2, rutin; (C) blank plasma; (D) 1, carbamazepine; 2, rhynochophylline; (E) blank plasma; (F) 1, phloroglucinol; 2, gastrodin.

shown in Fig. 1. No endogenous interference was observed at retention time of the analytes and internal standard.

Preparation of the solution for animal oral administration. Tiangou antihypertensive capsule powders were reconstituted in $0.5 \% \mathrm{CMC}-\mathrm{Na}$ solution at a concentration of $0.1 \mathrm{~g} / \mathrm{ml}$. HPLC was used to determine the content of baicalin, gastrodin and rhynochophylline in the Tiangou antihypertensive capsule. The data were used as a benchmark to prepare single herb extract solution. Baicalin and Baicalein standard were reconstituted in $0.5 \%$ CMC-Na solution at a concentration of $5.0 \mathrm{mg} / \mathrm{ml}$.

A low concentration of rhynochophylline in the samples or low sensitivity of the detector towards the rhynochophylline may explain the non-detection. For preparation of the extract, $10 \mathrm{~g}$ powder of the Tiangou antihypertensive capsule was extracted with methanol under sonication for $30 \mathrm{~min}$ and filtrated. The extracting solution was centrifuged at $500 \mathrm{x} \mathrm{g}$ for $10 \mathrm{~min}$ and dried using nitrogen. The residue was
Table V. Oral administration dosage.

\begin{tabular}{lcc}
\hline Group & $\begin{array}{c}\text { Aharpleaf uncaria stem } \\
\text { with Hooks extract }(\mathrm{ml})\end{array}$ & $\begin{array}{c}\text { Tiangou antihypertensive } \\
\text { capsule }(\mathrm{ml})\end{array}$ \\
\hline $\mathrm{H}$ & - & 0.6 \\
$\mathrm{I}$ & 0.6 & - \\
\hline
\end{tabular}

reconstituted in $0.5 \% \mathrm{CMC}-\mathrm{Na}$ solution at a concentration of $0.1 \mathrm{~g} / \mathrm{ml}$.

Oral administration. A total of 54 male SD rats with a body weight of $220 \pm 20 \mathrm{~g}$ were randomly divided into 9 groups. The rats were acclimatized for 5 days, and fasted with free access to water until $12 \mathrm{~h}$ prior to the experiment. The rats were administered orally with the abovementioned drug solutions, and the doses are shown in Tables III-V.

Collection of blood samples. After administration, the rats were anaesthetized with $10 \%$ Chlorali hydras. Samples were collected from the suborbital veniplex prior to administration and at 5, 10, 20, 30, 60, 90, 120, 180, 240, 360, 480, 600 and 720 min after administration. The plasma was placed into a tube in which there was heparin and was centrifuged at $850 \mathrm{x} \mathrm{g}$ for $10 \mathrm{~min}$. Supernatant $(0.2 \mathrm{ml})$ was transferred to a sample tube and stored at $-20^{\circ} \mathrm{C}$ in the refrigerator until analysis.

\section{Preparation of rat plasma sample}

Baicalin plasma treatment method. Plasma was defrosted at $25^{\circ} \mathrm{C}$, and $200 \mu \mathrm{l}$ of plasma sample was transferred into an Eppendorf tube. Subsequently, $50 \mu 1$ of rutin solution and $50 \mu 1$ of sodium dihydrogen phosphate solution $(1 \mathrm{~mol} / \mathrm{l})$ were added and vortex-mixed for $1 \mathrm{~min}$. Methanol $(100 \mu \mathrm{l})$ and acetonitrile (300 $\mu \mathrm{l})$ were added, vortex-mixed for $1 \mathrm{~min}$ and centrifuged at $850 \mathrm{x} \mathrm{g}$ for $10 \mathrm{~min}$ to precipiate protein. The supernatant was evaporated to dryness under a gentle stream of nitrogen at $25^{\circ} \mathrm{C}$. The residue was dissolved in $100 \mu 1$ mobile phase and filtered with a $0.22 \mu \mathrm{m}$ microporous membrane. Sample solution $(20 \mu \mathrm{l})$ was injected into the chromatographic system for analysis.

Gastrodin plasma treatment method. Plasma was defrosted at room temperature and $200 \mu \mathrm{l}$ of plasma sample was transferred into an Eppendorf tube. Subsequently, $100 \mu 1$ mobile 
Table VI. Pharmacokinetics parameters of baicalin with low, medium and high dose of Tiangou antihypertensive capsule ( $\mathrm{n}=6$ ).

\begin{tabular}{|c|c|c|c|c|}
\hline Parameter & Low dose & Medium dose & High dose & $\begin{array}{c}\text { Scutellaria baicalensi } \\
\text { extract }\end{array}$ \\
\hline $\mathrm{T}_{1 \max } / \mathrm{min}$ & $28.4 \pm 4.082$ & $28.3 \pm 3.174$ & $30.0 \pm 1.136$ & $28.6 \pm 2.798$ \\
\hline $\mathrm{T}_{2 \max } / \min$ & $280.0 \pm 21.967$ & $283.0 \pm 24.32$ & $300.0 \pm 45.92$ & $271 \pm 34.45$ \\
\hline $\mathrm{C}_{1 \max } / \mu \mathrm{g} / \mathrm{ml}$ & $2.223 \pm 0.441$ & $4.592 \pm 0.485$ & $6.906 \pm 0.475$ & $5.021 \pm 0.531$ \\
\hline $\mathrm{C}_{2 \max } / \mu \mathrm{g} / \mathrm{ml}$ & $1.799 \pm 0.176$ & $5.793 \pm 0.395$ & $7.89 \pm 1.407$ & $6.858 \pm 0.834$ \\
\hline $\mathrm{AUC}_{(0-\mathrm{t})} / \mu \mathrm{g} / \mathrm{ml} \cdot \mathrm{h}$ & $907.61 \pm 103.58$ & $2,194.84 \pm 153.38$ & $3,394.69 \pm 368.61$ & $2,201.32 \pm 156.41$ \\
\hline
\end{tabular}

AUC, area under the curve.



Figure 2. Mean plasma concentration-time curve of baicalin in rats after oral administration of different doses of Tiangou antihypertensive capsule.

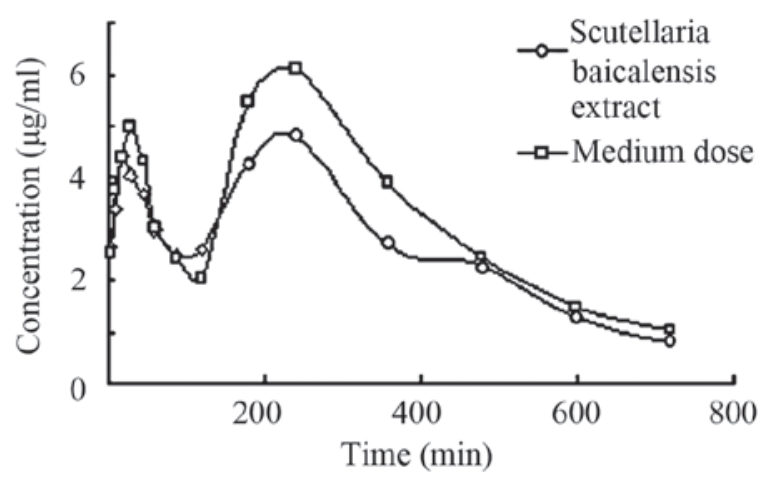

Figure 3. Mean plasma concentration-time curve of baicalin in rats after oral administration of extract and medium dose Tiangou antihypertensive capsule.

phase and $100 \mu \mathrm{l}$ phloroglucinol solution were added and the mixture was vortex-mixed for $1 \mathrm{~min}$. Acetonitrile $(1 \mathrm{ml})$ was then added, the mixture was vortex-mixed for $1 \mathrm{~min}$ and centrifuged at 9,000 x $\mathrm{g}$ for $10 \mathrm{~min}$ to precipitate the protein. The supernatant was dried under a gentle stream of nitrogen at $25^{\circ} \mathrm{C}$. Then, $200 \mu \mathrm{l}$ of mobile phase was used to dissolve the residue, which was centrifuged at $850 \mathrm{x}$ g for $10 \mathrm{~min}$, and a volume of $20 \mu \mathrm{l}$ sample solution was injected into the chromatographic system for analysis.

Rhynochophylline plasma treatment method. Plasma was defrosted at room temperature and $200 \mu \mathrm{l}$ of plasma sample was transferred into an Eppendorf tube. Carbamazepine

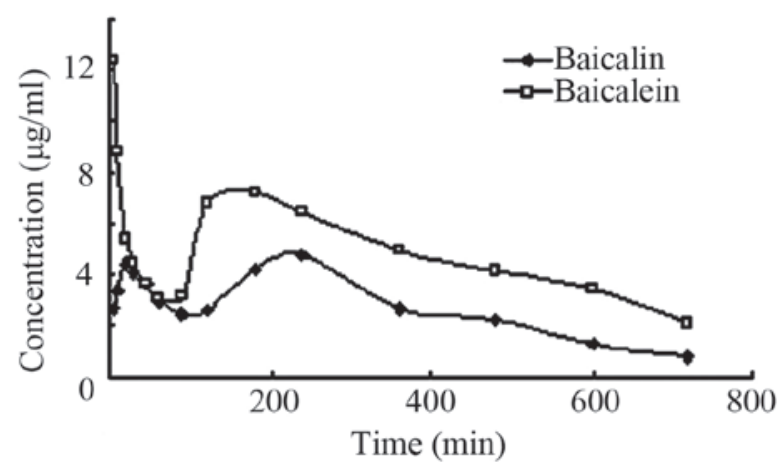

Figure 4. Concentration-time curve of baicalin after oral administration of baicalin and baicalein.

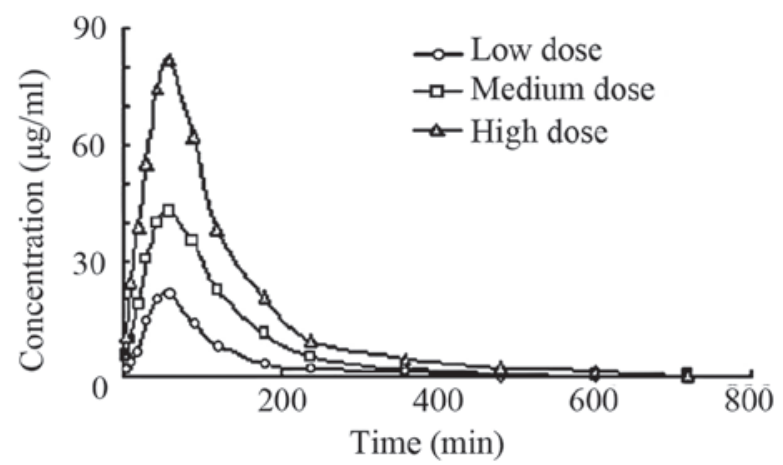

Figure 5. Mean plasma concentration-time curves of gastrodin in rats after ig Tiangou antihypertensive capsule at three dosage levels.

solution $(50 \mu \mathrm{l}), 200 \mu \mathrm{l}(0.1 \mathrm{~mol} / \mathrm{l}) \mathrm{NaOH}$ solution, and $1 \mathrm{ml}$ ether were added, the mixture was vortex-mixed for $3 \mathrm{~min}$ and centrifuged at $500 \mathrm{x} \mathrm{g}$ for $10 \mathrm{~min}$. The organic layer was then transferred into another tube and the above procedures were repeated. The organic phase was combined and dried under nitrogen. Methanol $(100 \mu \mathrm{l})$ was used to dissolve the residue which was centrifuged at $850 \mathrm{x}$ g for $10 \mathrm{~min}$, and then $20 \mu \mathrm{l}$ solution was injected into the chromatographic system for analysis.

The contents of three compounds in plasma were quantitatively determined by the above-mentioned analysis method. The concentration-time curve was then drawn and was used to analyze the absorption mechanism of such compounds. 
Table VII. Pharmacokinetics parameters of baicalin and baicalein groups $(n=6)$.

\begin{tabular}{lcc}
\hline & \multicolumn{2}{c}{ Group } \\
\cline { 2 - 3 } Parameter & Baicalin & Baicalein \\
\hline $\mathrm{T}_{1 \max } / \mathrm{min}$ & $26.573 \pm 1.372$ & $5.137 \pm 0.521$ \\
$\mathrm{~T}_{2 \max } / \min$ & $226.436 \pm 19.157$ & $187.331 \pm 34.452$
\end{tabular}



Figure 6. Mean plasma concentration-time curves of gastrodin in rats after ig Gastrodia Rhizoma extract and Tiangou antihypertensive capsule.

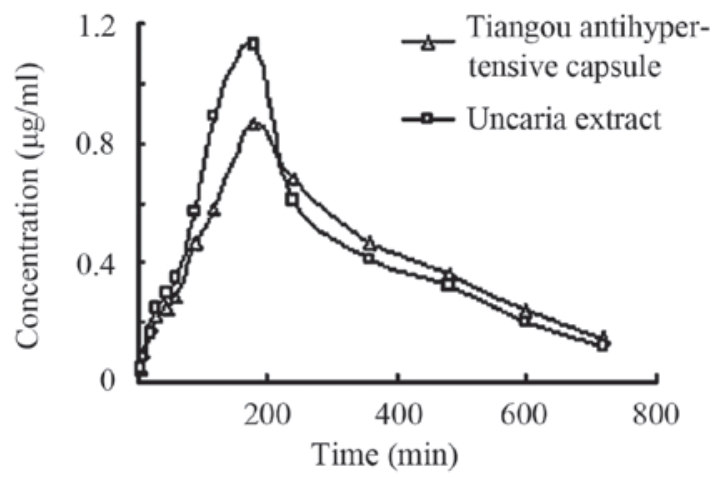

Figure 7. Concentration-time curve of rhynochophylline after oral administration of Tiangou antihypertensive capsule and Gambir plant extract.

\section{Results and Discussion}

Concentration-time curve and pharmacokinetic parameter of baicalin. The content of baicalin in groups A-D was determined by the abovementioned analysis method. The concentration-time curves of baicalin were drawn as follows: Time as $\mathrm{x}$-coordinate and the concentration of baicalin in plasma as y-coordinate. Fig. 2 shows the concentration-time curve of baicalin in rats after oral administration of a low-, medium- and high-dose of the Tiangou antihypertensive capsule. Fig. 3 shows the concentration-time curve of baicalin in plasma of rats after oral administration of the Scutellaria baicalensis extract and a medium dose of the Tiangou antihypertensive capsule.

As shown in Figs. 2 and 3, as well as in Table VI, no significant difference was found for the peak concentration time of baicalin between the groups. We obtained a dose-dependent plasma concentration profile as well as a peak concentration of baicalin. The results suggest that the absorption of baicalin was attributed to passive absorption. The peak concentrations of the medium-dose group were $4.592 \pm 0.485$ and $5.793 \pm 0.395 \mu \mathrm{g} / \mathrm{ml}$ and the peak concentration of the extract were $5.021 \pm 0.531$ and $6.858 \pm 0.834 \mu \mathrm{g} / \mathrm{ml}$. The t-test indicated no significant difference for the area under the curve (AUC) of baicalin between the medium-dose group and Scutellaria baicalensis extract group $(\mathrm{P}>0.05)$. These results showed that the Tiangou antihypertensive capsule did not influence baicalin absorption, but made the drug effect more mild, and maintained the plasma concentration of baicalin to avoid a peak-valley phenomenon.

The content of baicalin in groups $\mathrm{F}$ and $\mathrm{G}$ was determined by the above-mentioned analysis method. Fig. 4 and Table VI show that baicalin exhibited a bimodal phenomenon in the concentration-time curve, which was consistent with previous findings $(9,10)$. In the concentration-time profile of the baicalein group, the first peak occurred at $5 \mathrm{~min}$. The peak concentration was much greater than that of the baicalin group. Both of them exhibited a bimodal phenomenon in concentration-time profile.

Blood samples of rats did not contain any baicalein (11); nevertheless, an amount of baicalin was detected in the samples. The result of the experiment showed that baicalin has a high bioavailability and faster absorption. Previous findings indicate that the bacterium in intestinal was able to hydrolyze baicalin to baicalein (12). Only in this manner, could baicalin be absorbed and this metabolic process is an important stage for baicalin absorption (12). The intestinal absorption experiment showed that baicalin has a high absorption rate in duodenum and cecum (13). It may be a cause of the bimodal phenomenon after oral administration baicalin. As shown in Figs. 5-7, the concentration-time curve of gastrodin and rhynochophylline do not have a bimodal phenomenon. Therefore, the bimodal phenomenon caused by baicalin inhibition of gastric emptying may be excluded. By intravenous injection, the bimodal phenomenon was observed in the concentration-time curve. If rat biliary tract is ligated, the concentration-time curve does not observe the bimodal phenomenon. This phenomenon probably occurs due to distribution re-absorption and enterohepatic circulation. The present results therefore showed that, Tiangou antihypertensive capsule did not have any effect on baicalin absorption, making the drug effect more mild and maintaining the concentration of baicalin in vivo more steady. These findings are consistent with those of previous investigation (14).

Concentration-time curve and pharmacokinetic parameter of gastrodin. The content of gastrodin in groups A, B, C and E was determined by the abovementioned analysis method. The concentration-time curves of gastrodin were drawn as follows: Time as $\mathrm{x}$-coordinate and the concentration of gastrodin in plasma as $y$-coordinate. Fig. 3 shows the concentration-time curve of gastrodin after oral administration of low, medium and high dose of Tiangou antihypertensive capsule. Fig. 5 shows the concentration-time curve of gastrodin after oral administration of the Gastrodia Rhizoma extract and medium dose of the Tiangou antihypertensive capsule. 
Table VIII. Pharmacokinetic parameters of gastrodin after ig three dosage levels of Tiangou antihypertensive capsule and Gastrodia Rhizoma extract.

\begin{tabular}{|c|c|c|c|c|}
\hline \multirow[b]{2}{*}{ Parameter } & \multicolumn{3}{|c|}{ Dose } & \multirow[b]{2}{*}{ Extract } \\
\hline & Low dose & Mid & High & \\
\hline $\mathrm{K}_{\mathrm{e}} / \min$ & $0.017 \pm 0.001$ & $0.0151 \pm 0.003$ & $0.013 \pm 0.001$ & $0.0204 \pm 0.004$ \\
\hline $\mathrm{K}_{\mathrm{a}} / \min$ & $0.032 \pm 0.003$ & $0.035 \pm 0.005$ & $0.031 \pm 0.007$ & $0.036 \pm 0.003$ \\
\hline $\mathrm{T}_{\max } / \mathrm{min}$ & $42.005 \pm 2.918$ & $43.971 \pm 2.973$ & $47.673 \pm 4.537$ & $36.670 \pm 3.098$ \\
\hline $\mathrm{AUC} / \mu \mathrm{g} \cdot \mathrm{ml}^{-1} \cdot \min$ & $2,263.1 \pm 411.7$ & $5,187.2 \pm 871.5$ & $10,261 \pm 354.2$ & $5,462.1 \pm 281.2$ \\
\hline $\mathrm{V} / \mathrm{ml} \cdot \mathrm{mg}^{-1}$ & $0.225 \pm 0.033$ & $0.223 \pm 0.041$ & $0.248 \pm 0.037$ & $0.161 \pm 0.029$ \\
\hline
\end{tabular}

AUC, area under the curve.

Table IX. Pharmacokinetic parameters of rhynochophylline in Tiangou antihypertensive capsule and Gambir plant extract $(n=6)$.

\begin{tabular}{lcc}
\hline Parameter & $\begin{array}{c}\text { Tiangou antihypertensive } \\
\text { capsule }\end{array}$ & $\begin{array}{c}\text { Gambir plant } \\
\text { extract }\end{array}$ \\
\hline $\mathrm{K}_{\mathrm{e}} / \mathrm{h}$ & $0.2643 \pm 0.0132$ & $0.2909 \pm 0.028$ \\
$\mathrm{~K}_{\mathrm{a}} / \mathrm{h}$ & $0.4965 \pm 0.0438$ & $0.6274 \pm 0.1528$ \\
$\mathrm{~T}_{\max } / \mathrm{h}$ & $2.7242 \pm 0.1282$ & $2.3994 \pm 0.2295$ \\
$\mathrm{AUC} / \mu \mathrm{g} / \mathrm{ml} / \mathrm{h}$ & $5.1222 \pm 0.7919$ & $5.1432 \pm 0.4537$ \\
$\mathrm{~V} / \mathrm{ml} / \mathrm{ml}$ & $13.5863 \pm 0.7832$ & $11.1187 \pm 0.8734$ \\
\hline
\end{tabular}

AUC, area under the curve.

Figs. 5 and 6, and Table VIII show that, AUC and $\mathrm{C}_{\max }$ were proportional to the dose administered. Compared with the Gastrodia Rhizoma extract, $\mathrm{C}_{\max }$ and $\mathrm{K}_{\mathrm{e}}$ of gastrodin in the Tiangou antihypertensive capsule groups were decreased while the Vd was significantly increased. However, no significant difference was found for AUC of gastrodin between the two groups.

Gastrodin in rats is consistent with a one-compartment model $(15,16)$. The peak of gastrodin occurred within $45 \mathrm{~min}$, and rapid decay occurred after $1 \mathrm{~h}$, indicating that gastrodin was not stored in the body, has a high absorption speed in the intestinal tract and exerts a rapid effect. The results demonstrated that the complex prescription exerted a certain effect on the absorption, distribution and elimination of gastrodin. The complex prescription delayed the absorption and excretion of gastrodin, prolonged the residence time of gastrodin in vivo, and could promote the distribution of gastrodin in vivo, but it did not have significant influence on bioavailability.

Concentration-time curve of rhynochophylline. As the data show in Table IX, compared with the Uncaria rhynchophylla extract group, the $\mathrm{K}_{\mathrm{a}}$ and $\mathrm{K}_{\mathrm{e}}$ of rhynochophylline were significantly decreased in the complex prescription group. However, there was effect on AUC. By contrast, the Vd of the Tiangou antihypertensive capsule group was significantly increased.
In consideration of the low content of rhynchophylline in powder and extract (17), we should set up a single experimental group. Summing up the above, on the premise of without influence drug absorption, complex prescriptions could avoid peak-valley phenomenon during taking medicine. And make the plasma concentratioin more smoothly. Comparison of the $\mathrm{Vd}$ we found Tiangou antihypertensive capsule could promote the in vivo distribution of rhynochophylline.

In conclusion, the results of the present study have shown that, the Tiangou antihypertensive capsule made the plasma concentration of effective components more stable. It was consistent with the medication principle of smooth reduction. Complex prescription did not have a significant influence on the total absorption rate of single composition, respectively. The peak concentration of gastrodin occurred at an early stage, the peak concentration of rhynochophylline and baicalin occur subsequently, and the $t_{\max }$ of rhynochophylline is close to the $t_{\max }$ of baicalin. It may be associated with the pharmacological action of the synergistic antihypertensive effect, which remains to be investigated in future studies.

\section{References}

1. Wang YL, Li LJ and Zhang W: In vivo pharmacokinetics of paeonol from Tiangou Jiangya capsule in rats. Chin Exper Trad Med Formulae 18: 114-116, 2012.

2. Lou HW, Li LJ and Li J: Studies on absorption kinetics of rhynchophylline and isorhynchophylline of Tiangou Jiangya capsules in rats intestinal. Chin Exper Trad Med Formulae 19: 150-153, 2013.

3. Hu KJ, Wang YH and Wang D: The inhibited effect of chlorogenic acid from the honeysuckle on virus in vitro. Information Trad Chin Med 27: 27-28, 2010.

4. Hou Y, Zhu X and Cheng G: Study on the anti inflammatory mechanism of baicalin. J Acta Pharmacol Sin 35: 161-164, 2010.

5. Xiong Y, Fu YY, Kuang NZ, et al: Study on activity and mechanism of baical in against candida albicans. Chin Pharmacol Bull 20: 1404-1407, 2004.

6. Lin L, Liu JX, Zhang Y, Lin CR and Li LQ: Effect of different combinations of compound Danshen preparation on pharmacokinetic parameters of salvianolic acid B in rat plasma. Zhongguo Zhong Yao Za Zhi 33: 2683-2687, 2008 (In Chinese).

7. National Pharmacopoeia Committee: Pharmacopoeia of the People's Republic of China. Chemical Industry Press, Beijing, p301, 2015.

8. Wang J, Liu ZH, Long Y and Xin LN: HPLC determination of rhynchophylline and isorhynchophylline in Anshen Yangxue oral liquid. Zhong Yao Cai 32: 1134-1137, 2009 (In Chinese). 
9. Zhang J, Zhang S, Teng S and Zhai L: An LC-MS/MS method for simultaneous determination of four flavonoids from semen oroxyli in rat plasma and its application to a pharmacokinetic study. J Chromatogr B Analyt Technol Biomed Life Sci 1020 96-102, 2016

10. Tong L, Wan M, Zhang L, Zhu Y, Sun H and Bi K: Simultaneous determination of baicalin, wogonoside, baicalein, wogonin, oroxylin A and chrysin of Radix scutellariae extract in rat plasma by liquid chromatography tandem mass spectrometry. $\mathrm{J}$ Pharm Biomed Anal 70: 6-12, 2012.

11. Chen H, Li Z, Li YJ, Wu XW, Wang SR, Chen K, Zheng XX, Du Q and Tang D: Simultaneous determination of baicalin, oroxylin A-7-O-glucuronide and wogonoside in rat plasma by UPLC-DAD and its application in pharmacokinetics of pure baicalin, Radix Scutellariae and Yinhuang granule. Biomed Chromatogr 29: 1819-1825, 2015.

12. Noh K, Kang Y, Nepal MR, Jeong KS, Oh G, Kang MJ, Lee S, Kang W, Jeong HG and Jeong TC: Role of intestinal microbiota in baicalin-induced drug interaction and its pharmacokinetics. Molecules 21: 337-340, 2016
13. Li LJ, Li J and Lou HW: Study on in situ intestinal absorption of baicalin contained in Tiangou Jiangya capsules. Zhongguo Zhong Yao Za Zhi 38: 894-898, 2013 (In Chinese).

14. Xiao L, Wang F, Li HD and Zhao XY: Pharmacokinetic study on baicalin of Qingkailing injection in rats. Zhongguo Zhong Yao Za Zhi 32: 2534-2538, 2007 (In Chinese).

15. Cai Z, Hou S, Li Y, Zhao B, Yang Z, Xu S and Pu J: Effect of borneol on the distribution of gastrodin to the brain in mice via oral administration. J Drug Target 16: 178-184, 2008.

16. Li LJ and Yan R: Pharmacokinetics of gastrodin from Tiangou Jiangya capsule in rats. J. Zhongguo Zhong Yao Za Zhi 39: 2964-2967, 2014 (In Chinese).

17. Ge Z, Xie Y, Liang Q, Wang Y and Luo G: Pharmacokinetic Comparative Study of Gastrodin and Rhynchophylline after Oral Administration of Different Prescriptions of Yizhi Tablets in Rats by an HPLC-ESI/MS Method. Evid Based Complement Alternat Med 2014: 167253, 2014. 